\title{
On the influence of magnetic fields in neutral planetary wakes
}

\author{
C. Villarreal D'Angelo ${ }^{1}$, M. Schneiter ${ }^{1}$ and A. Esquivel ${ }^{2}$ \\ ${ }^{1}$ Instituto de Astronomía Teórica y Experimental, Conicet-UNC, \\ Laprida 854, X5000BGR, Córdoba, Argentina \\ email: carolina.villarreal@unc.edu.ar \\ ${ }^{2}$ Instituto de Ciencias Nucleares, UNAM, \\ C. Univ., AP 70-543 México D.F., México
}

\begin{abstract}
We present a 3D magnetohydrodynamic study of the effect that stellar and planetary magnetic fields have on the calculated Ly $\alpha$ absorption during the planetary transit, employing parameters that resemble the exoplanet HD209458b. We assume a dipolar magnetic field for both the star and the planet, and use the Parker solution to initialize the stellar wind. We also consider the radiative processes and the radiation pressure.

We use the numerical MHD code GuACHO to run several models varying the values of the planetary and stellar magnetic moments within the range reported in the literature.

We found that the presence of magnetic fields influences the escaping neutral planetary material spreading the absorption $\mathrm{Ly} \alpha$ line for large stellar magnetic fields.
\end{abstract}

Keywords. line: profiles, methods: numerical, magnetic fields, stars: winds, stars: individual (HD209458).

\section{Introduction}

Despite the large number of Hot Jupiter discovered, only a few of them have observations that indicates that they are losing neutral material from their atmospheres. The first one of this cases was HD 209458b (Vidal-Madjar et al. 2003). The Ly $\alpha$ observations analysed by Vidal-Madjar et al. (2003) showed an extra absorption during the planetary transit that, together with the detection of heavier elements (Vidal-Madjar et al. (2004, 2013); Ben-Jaffel \& Sona Hosseini (2010)) in the upper atmosphere, confirmed the "blow off" state of the atmosphere. Later, similar observation were made for HD 189733b (Lecavelier Des Etangs et al. (2010); Bourrier et al. (2013)), 55 Cnc b (Ehrenreich et al. (2012)) and GJ 436b (Kulow et al. (2014); Ehrenreich et al. (2015)).

In transit, the Ly $\alpha$ profile of HD 209458b show an absorption of approximately 10 per cent in the line wings, at $\pm 100 \mathrm{~km} \mathrm{~s}^{-1}$, with absorptions at higher velocities in the blue side. To explain this observational behaviour several models have been proposed. Tremblin \& Chiang (2013) studied the charge exchange mechanism together with the interaction region between the winds with $2 \mathrm{D}$ hydrodynamic models. Schneiter et al. (2016) studied the influence of the photoionization process in the interaction of the winds using a hydrodynamic code. All the models in this work where able to reproduce the absorption in the blue part of the Ly $\alpha$ line, failing in reproducing the absorption in the red part.

A logic step towards a self consistent model is to include the magnetic fields of both the star and the planet. For the star, the most straight forward assumption is to consider it as a solar analogue. For the planet, several works agree that the value of magnetic dipole moment is of the order of $0.1 \mu_{J}$, with $\mu_{J}=1.56 \times 10^{26} \mathrm{~A} \mathrm{~m}^{2}$ the magnetic moment of Jupiter (Durand-Manterola 2009; Sánchez-Lavega 2004; Khodachenko et al. 2012, 2015; 


\begin{tabular}{ll}
\hline Stellar parameters & Value \\
\hline Radius & $1.2 \mathrm{R}_{\odot}$ \\
Mass & $1.1 \mathrm{M} \odot$ \\
$r_{w s}$ & $R_{*}$ \\
$T_{*}\left(r_{w}\right)$ & $1.56 \times 10^{6} \mathrm{~K}^{\mathbf{a}}$ \\
$\rho\left(r_{w}\right)$ & $1.544 \times 10^{-16} \mathrm{~g} \mathrm{~cm}^{-3} \mathrm{a}$ \\
$\log L_{E U V}$ & $<27.74 \mathrm{erg} \mathrm{s}^{-1} \mathbf{~ b}$ \\
Ionizing photon rate & $2.4 \times 10^{38} \mathrm{~s}^{-1}$ \\
\hline Planetary parameters & Value \\
\hline Radius & $1.3 \mathrm{R}_{J u p}$ \\
Mass & $0.6 \mathrm{M}_{J u p}$ \\
$a$ & $0.047 \mathrm{AU}$ \\
Orbital period & $3.52 \mathrm{day}$ \\
$r_{w p}$ & $3 R_{p}$ \\
$T_{p}\left(r_{w}\right)$ & $1 \times 10^{4} \mathrm{~K}^{\mathbf{c}}$ \\
$v_{p}\left(r_{w}\right)$ & $10 \mathrm{~km} \mathrm{~s}^{-1} \mathbf{c}$ \\
$\dot{M}_{p}$ & $1 \times 10^{10} \mathrm{~g} \mathrm{~s}^{-1} \mathbf{c}$ \\
\hline
\end{tabular}

Table 1. Stellar and planetary wind parameters used in the simulations. ${ }^{a}$ Vidotto et al. (2009), ${ }^{b}$ Sanz-Forcada et al. (2011), ${ }^{c}$ Murray-Clay et al. (2009).

Kislyakova et al. 2014). But, higher values have been used (Owen \& Adams 2014; Vidotto et al. 2015). The lack of observational constraints made this an open debate. In this work, we assume that $\mu_{p}$ is in the range of $\left.[0.2-1.2] \mu_{J}\right)$.

The current work is an effort to study the effect that different magnetic field moments (stellar and planetary) might have on the Ly $\alpha$ absorption profile.

\section{Numerical models}

The interaction between the planet and stellar winds was modelled with the 3D/MHDradiative code GUACHO, in a Cartesian mesh of 256x128x256 cells, with a resolution of $5 \times 10^{-4}$ au.

The code evolves the ideal MHD equations together with an extra equation that tracks the neutral density in each cell, taking into account the process of recombination, collisional ionization and photoionization of the gas.

The model includes the gravitational forces of the planet and the star, together with the radiation pressure implemented as a reduction of the stellar gravity on the neutral planetary material. A full explanation of the model is given in Schneiter et al. (2016).

In the numerical models, the star is at the center of the computational domain with the planet orbiting around it in a circular orbit. The ionized stellar wind is launched at the stellar radius, and it is initialized with the parameters used in the work of Pneuman \& Kopp (1971) for an isothermal wind. The value of the polytropic index is set to $\gamma=1.01$ for the stellar wind base but is allow to varies as a function of the neutral fraction.

The star has a magnetic dipole oriented perpendicular to the orbital plane. The values of the stellar magnetic dipole, $B_{*},([1-5]$ Gauss) are taken from Vidotto et al. (2009). To take into account the photoionization process we divide the EUV stellar flux into $10^{7}$ photon packages. This packages are launched from the surface of the star at random position in random directions. This is the same approach used in the work of Schneiter et al. (2016). 


\begin{tabular}{lll}
\hline Model & $B_{*}[\mathrm{G}]$ & $B_{p}[\mathrm{G}]$ \\
\hline $\mathrm{A} 1$ & 1 & 1 \\
$\mathrm{~A} 2$ & 1 & 5 \\
$\mathrm{~B} 1$ & 5 & 1 \\
$\mathrm{~B} 2$ & 5 & 5 \\
\hline
\end{tabular}

Table 2. Model parameters.

We use the planetary parameters of HD 209458b. The planetary wind is launched at $3 R_{p}$ with a density, temperature and velocity values taken from the work of MurrayClay et al. (2009). As in the previous work (Schneiter et al. 2016), we assume that the escaping atmosphere of the planet is composed of Hydrogen and at the base of the wind it remains $20 \%$ neutral. The mass loss rate of the planet is assumed to be $\dot{M}_{p}=1 \times 10^{10}$ $\mathrm{g} \mathrm{s}^{-1}$ based on the results of Schneiter et al. (2007); Villarreal D'Angelo et al. (2014); Schneiter et al. (2016). This value of $\dot{M}_{p}$, together with the value of stellar $F_{E U V}$ used, gives a heating efficiency (Yelle 2004) at the wind base of 0.2 .

The planetary magnetic field, $B_{p}$ is assumed dipolar with it's axis oriented perpendicular to the orbital plane and in the same direction as the magnetic field of the star. The values used for the planetary magnetic field are [1,5] Gauss at the poles, extrapolated at the radius where the wind is launched.

The initial parameters in the simulations are shown in the Table 1.

To simplify the analysis we decided to run varying only $B_{*} \& B_{p}$. The models are introduce in Table 2.

\section{Results \& Discussion}

The global behaviour of the numerical models show that a cometary tail is formed trailing the planet. As depicted on the left panel of figure (1), this tail can be divided in two parts, one of completely ionized material (farther away from the planet), as a result of the interaction with both the stellar wind and the EUV flux, and another with partially neutral material (closer to the planet). Around to the planet, these partially neutral cloud is composed of atmospheric material and stellar wind particles that became neutralized when interact with the planetary wind. This neutral material is the one responsible for the absorption in the $\operatorname{Ly} \alpha$ line.

The right panel of figure (1) show the temperature contour in the orbital plane. The variation of the polytropic index with the neutral fraction is only important around the planet. Hence, the temperature of the stellar wind shows no significant variation except for the interaction region, where the planetary material gets heated and the stellar wind cooled at $T \sim 10^{5} \mathrm{~K}$.

In order to reproduce the $\operatorname{Ly} \alpha$ observations we computed the absorption profile in the same manner as in Schneiter et al. (2007); Villarreal D'Angelo et al. (2014) and Schneiter et al. (2016). In figure (2) we can see that the resulting line profile is asymmetric for both models, i.e there is more absorption in the blue side than in the red side, as in the observations. The models show a $10 \%$ of absorption in the blue wing, corresponding to velocities close to $-100 \mathrm{~km} \mathrm{~s}^{-1}$ for models A1 \& A2, reaching $-200 \mathrm{~km} \mathrm{~s}^{-1}$ for the models B1 \& B2. As mentioned earlier, four different models were tested, two planetary magnetic field values $([1,5] \mathrm{G})$ for each stellar magnetic field $([1,5] \mathrm{G})$. As observed in the Ly $\alpha$ profiles of figure (2), the change in planetary magnetic field has almost none influence on the absorption. On the other hand, increasing the stellar wind magnetic field magnitude widens the line profile. In the wings of the profile, the models with higher 

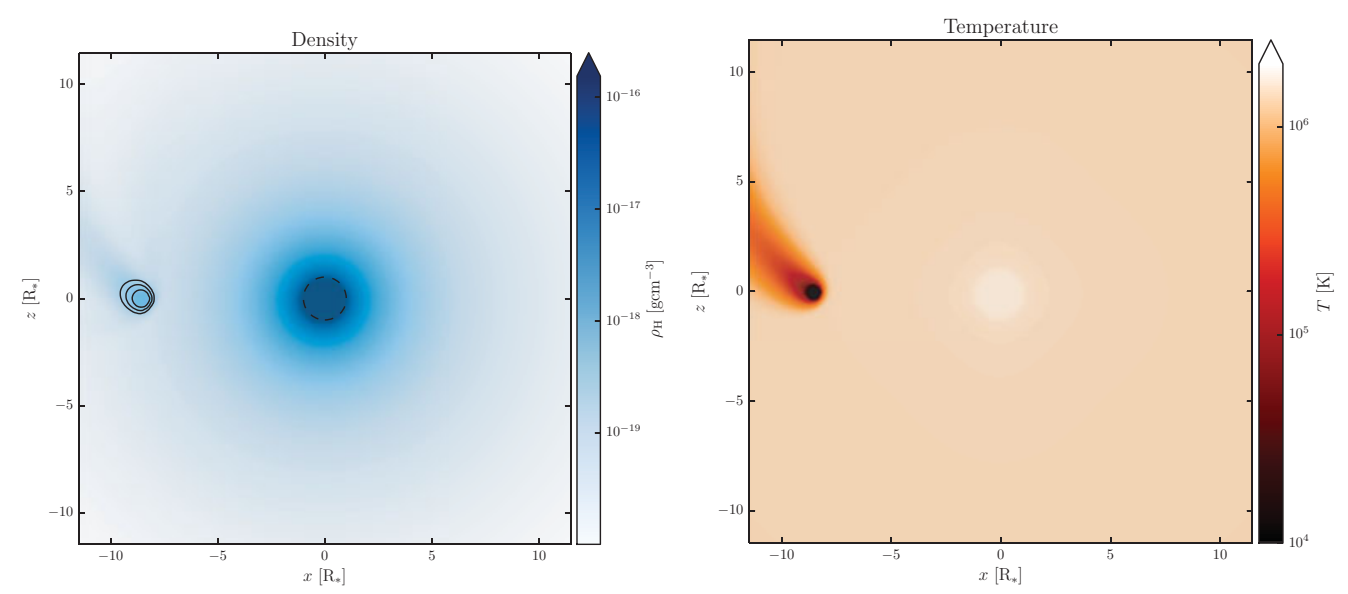

Figure 1. Contour of density and temperature in the orbital plane for model A1. In the left panel, the black contour lines around the planet correspond to the value $[0.9,0.99,0.999]$ of the ionization fraction and the dashed black line is the base of the stellar wind at the stellar radius.

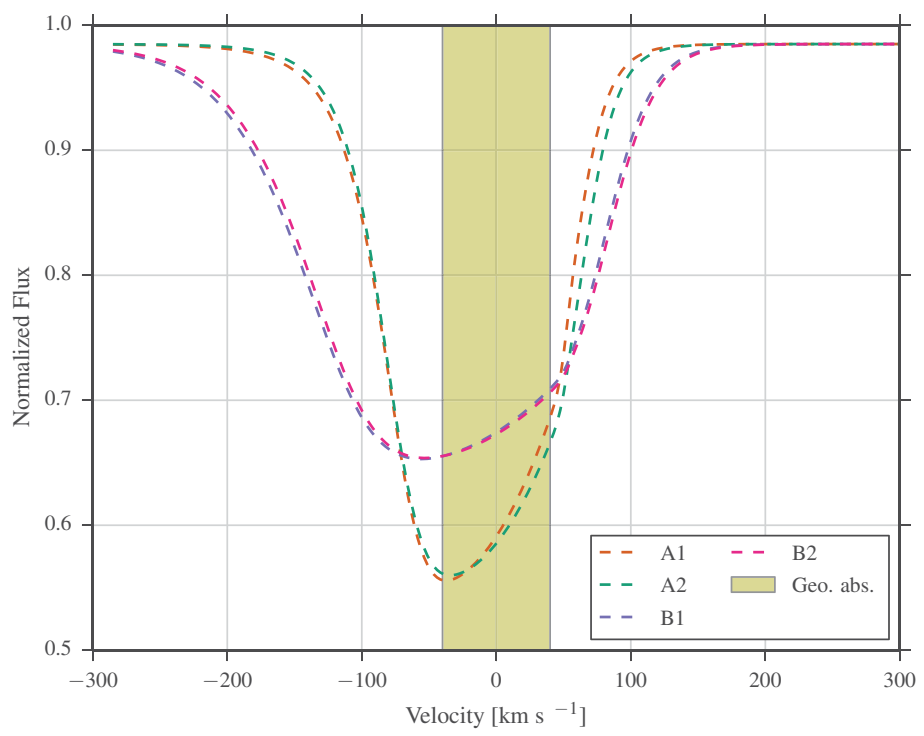

Figure 2. Normalized stellar emission as a function of the line of sight velocity in the Ly $\alpha$ line. The yellow stripe correspond to part of the line contaminated with the geocoronal glow, omitted in the total absorption calculations.

stellar magnetic field $(5 \mathrm{G})$ produce more absorption than the models with $1 \mathrm{G}$. Given the stellar magnetic field structure, the higher the magnetic pressure $\left(P_{m a g}=B^{2} / 8 \pi\right)$, the higher the compression of the planetary material on the poles. This compression produces an enhanced acceleration mainly towards the tail, in the direction of the magnetic field lines (see right panel of figure 3), but also, less pronounced in the direction towards the host star. $B_{*}$ is also responsible for the enhancement of the neutral density for the more compressed case.

For models A1 \& A2, the partially neutral cloud surrounding the planet has a more prolate shape with neutrals farther away from the star. This is depicted in figure (4), 

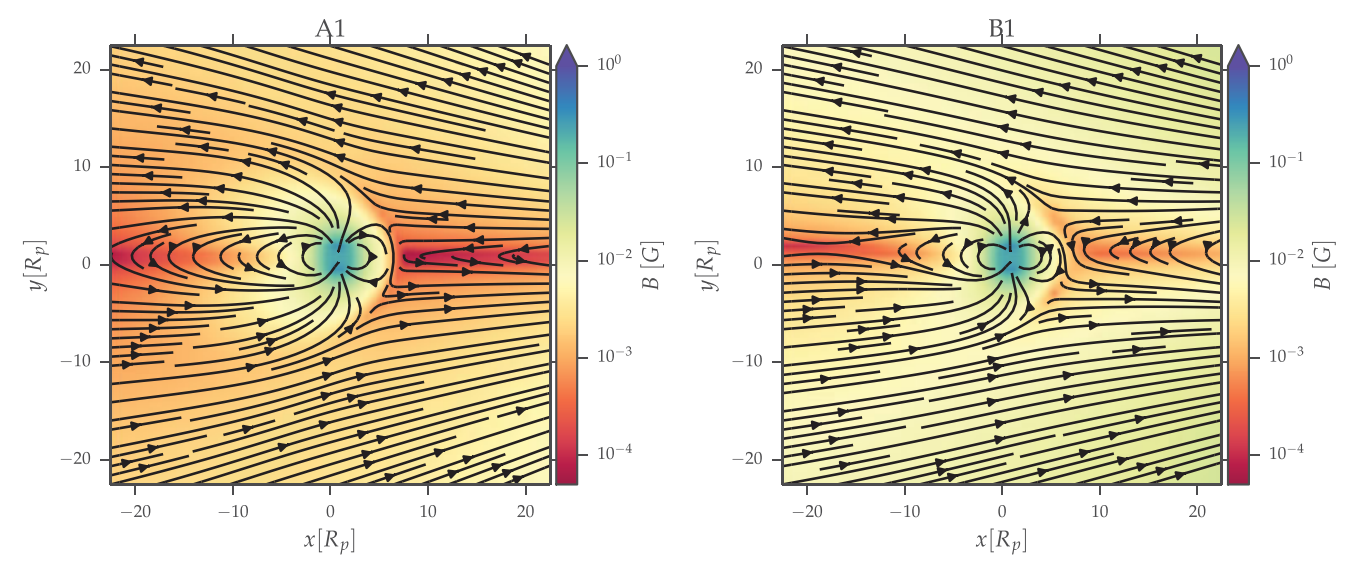

Figure 3. Magnetic field lines and total magnetic field value for models $A 1$ and $B 1$ in the vicinity of the planet position in a plane perpendicular to the orbital plane.
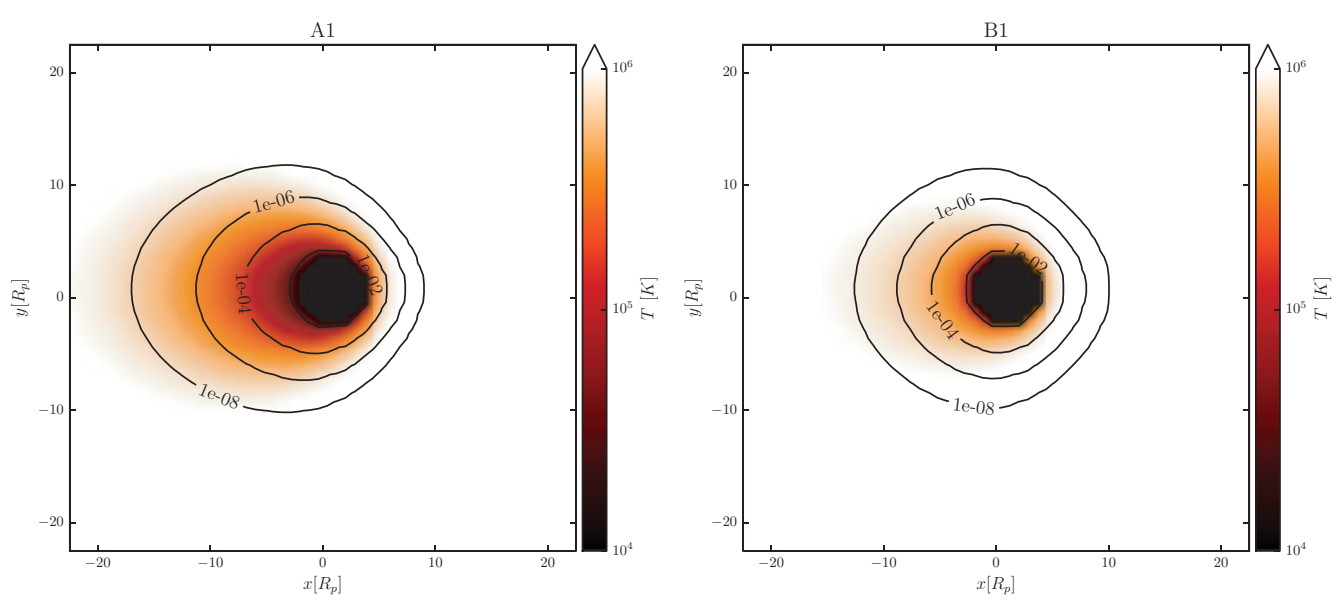

Figure 4. Temperature map, in the plane perpendicular to the orbital plane, with contours of the neutral material normalized to the total density of the planet $\left(\rho_{p}=9.2 \times 10^{-18} \mathrm{~g} \mathrm{~cm}^{-3}\right)$ for models A1 and B1.

where contours of the neutral density, normalized to the planetary density, are show around the planet.

As stated before, the shape of this cloud is modelled by the total pressure of the stellar wind. When the magnetic stellar pressure at the poles compresses the planetary wind (models B1 \& B2), it enhances his neutral density and temperature, allowing the planetary wind to balance the total pressure of the stellar wind farther away from the planet towards the star. The neutral material that escapes to the tail is ionized much faster in this case due to the higher temperature.

For cases A1 \& A2, the magnetic stellar compression is lower, hence, the partially neutral cloud can expand a bit more away from the planet. However, the balance between the planetary and the stellar pressures take place closer to the planet since this neutral cloud is now less denser and cooler (see figure (4)). 


\section{Summary}

A study of the influence of both the stellar and planetary magnetic fields on the Ly $\alpha$ absorption profile, based on a full 3D magnetohydrodynamic + photoionization model was introduced. From this study we were able to establish, within the range of parameters proposed, that the planetary magnetic field has no important imprint on the Ly $\alpha$ line, whereas the stellar magnetic field has a great influence on the blue part of the line, and a more subtle influence on the red part. Moreover, we were able to produce absorption at velocities below $-100 \mathrm{~km} \mathrm{~s}^{-1}$ with models with a magnetic field of $5 \mathrm{G}$. This absorption are the result of neutrals originated from recombination of stellar wind ions. As stated in Khodachenko et al. (2015), the higher the planetary magnetic field the lower the planetary mass loss rate. According to this work, the mass loss rate corresponding to $B_{p}=5 \mathrm{G}$ is an order of magnitude lower than the one used here. A lower value of $\dot{M}_{p}$ would help to distinguish the absorption profile between the models.

\section{References}

Ben-Jaffel, L. \& Sona Hosseini, S. 2010, ApJ, 709, 1284

Bourrier, V., Lecavelier des Etangs, A., Dupuy, H., et al. 2013, A\&A, 551, A63

Durand-Manterola, H. J. 2009, Plan. \& Space Sci., 57, 1405

Ehrenreich, D., Bourrier, V., Bonfils, X., et al. 2012, A\& A, 547, A18

Ehrenreich, D., Bourrier, V., Wheatley, P. J., et al. 2015, Nature, 522, 459

Khodachenko, M. L., Alexeev, I., Belenkaya, E., et al. 2012, ApJ, 744, 70

Khodachenko, M. L., Shaikhislamov, I. F., Lammer, H., \& Prokopov, P. A. 2015, ApJ, 813, 50

Kislyakova, K. G., Holmström, M., Lammer, H., Odert, P., \& Khodachenko, M. L. 2014, Science, 346,981

Kulow, J. R., France, K., Linsky, J., \& Loyd, R. O. P. 2014, ApJ, 786, 132

Lecavelier Des Etangs, A., Ehrenreich, D., Vidal-Madjar, A., et al. 2010, A\&A, 514, A72

Murray-Clay, R. A., Chiang, E. I., \& Murray, N. 2009, ApJ, 693, 23

Owen, J. E. \& Adams, F. C. 2014, MNRAS, 444, 3761

Pneuman, G. W. \& Kopp, R. A. 1971, Sol. Phys., 18, 258

Sanz-Forcada, J., Micela, G., Ribas, I., et al. 2011, A\&A, 532, A6

Sánchez-Lavega, A. 2004, ApJL, 609, L87

Schneiter, E. M., Velázquez, P. F., Esquivel, A., Raga, A. C., \& Blanco-Cano, X. 2007, ApJ, 671, L57

Schneiter, E. M., Esquivel, A., D'Angelo, C. S. V., et al. 2016, MNRAS, 457, 1666

Tremblin, P. \& Chiang, E. 2013, MNRAS, 428, 2565

Vidotto, A. A., Opher, M., Jatenco-Pereira, V., \& Gombosi, T. I. 2009, ApJ, 699, 441

Vidotto, A. A., Fares, R., Jardine, M., Moutou, C., \& Donati, J.-F. 2015, MNRAS, 449, 4117

Vidal-Madjar, A., Lecavelier des Etangs, A., Désert, J.-M., et al. 2003, Nature, 422, 143

Vidal-Madjar, A., Désert, J.-M., Lecavelier des Etangs, A., et al. 2004, ApJ, 604, L69

Vidal-Madjar, A., Huitson, C. M., Bourrier, V., et al. 2013, A\&A, 560, A54

Villarreal D'Angelo, C., Schneiter, M., Costa, A., et al. 2014, MNRAS, 438, 1654

Yelle, R. V. 2004, Icarus, 170, 167 